\title{
Estimating shear properties of walnut wood: a combined experimental and theoretical approach
}

\author{
Erik Valentine Bachtiar 1 - Markus Rüggeberg $\cdot$ Stefan Hering • \\ Michael Kaliske $\cdot$ Peter Niemz
}

Received: 5 July 2017/ Accepted: 20 November 2017 / Published online: 25 November 2017

(C) RILEM 2017

\begin{abstract}
In this study, several theoretical models to numerically estimate shear properties of orthotropic materials are introduced. These approaches are based on the combination of Hankinson's empirically derived formula with other empirical and analytical calculations. Next to shear moduli, which are estimated from the elastic moduli and Poisson's ratios, shear strengths are also estimated from the in-axis strengths. The models are validated by mechanical tests on walnut wood (Juglans regia L.), for which a
\end{abstract}

Electronic supplementary material The online version of this article (https://doi.org/10.1617/s11527-017-1119-2) contains supplementary material, which is available to authorized users.

E. V. Bachtiar $(\bowtie) \cdot$ M. Rüggeberg · P. Niemz Institute for Building Materials, ETH Zürich, 8093 Zurich, Switzerland

e-mail: berik@ethz.ch

\section{Rüggeberg}

Laboratory of Applied Wood Materials, Empa, 8600 Dübendorf, Switzerland

\section{S. Hering}

Former Institute for Building Materials, ETH Zürich, 8093 Zurich, Switzerland

\section{Kaliske}

Institute for Structural Analysis, Technische Universität Dresden, 01069 Dresden, Germany sufficient data set can be found in literature. The Arcan test is used to estimate the shear moduli, while the shear block test is used to estimate the shear strengths. The results show that the model, which is based on a combined use of Hankinson's formula and tensor rotation, gives the best estimation of shear moduli as evaluated by the minimum differences to the experimentally obtained results. For the shear strengths, a combination of Hankinson's formula and Norris' failure criterion shows the best agreement in comparison to the experimental data. The theoretical calculations may be used for a time efficient estimation of shear modulus and strength in comparison to the very time-consuming experimental estimation.

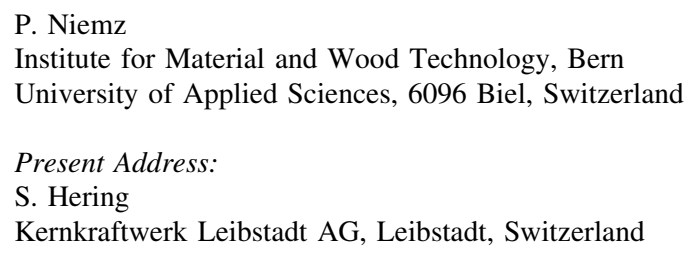


Keywords Approximation methods for orthotropic shear property · Hankinson's formula - Shear moduli . Shear strengths · Walnut wood (Juglans regia L.)

\section{Introduction}

Shear modulus and strength are important parameters for characterising the mechanical behaviour of a material. For isotropic materials, shear modulus $(G)$ can be analytically derived from the elastic modulus $(E)$ and the Poisson's ratio $(v)$ [1]. The shear strength $\left(\tau_{\mathrm{u}}\right)$ also can be empirically derived from the ultimate strength $\left(\sigma_{\mathrm{u}}\right)$ depending on the chosen failure criterion [2,3]. Yet, for orthotropic materials, such as wood, neither shear modulus nor shear strength can be derived from the other parameters. Specific experimental tests have been conducted to estimate these properties. However, it is extremely difficult to achieve pure shear loading. Shear stress is usually introduced by a pair of opposing normal loads with an equal magnitude and a slight offset. The rotation effect introduced by the offset of the loading is hereby ignored. Using this basis, the Iosipescu test [4] and the Arcan test $[5,6]$ are commonly used to determine the shear moduli. The shear block test [7] is used to investigate the shear strength parallel to the fiber. Unfortunately, due to the high shear resistance across the fiber, pure shear failure cannot be achieved. Performing the shear block test across the fiber nearly always result in compressive failure [8]. Other than these destructive methods, non-destructive methods, e.g. ultrasound technique [9], can also be used for determining the shear moduli, yet not the shear strengths.

Next to these methodological shortcomings of the mechanical tests, it requires tedious experimental work to investigate all six shear planes of an orthotropic material. In the case of wood with a natural variability in its properties and with the additional influence of moisture content on the mechanical properties, the experimental work is very time-consuming due to the large amount of samples that have to be tested and evaluated. The sample preparation, particularly for the v-notched specimens used in the Iosipescu and Arcan test, also requires a high degree of accuracy. The inaccuracies in the sample preparation combined with the non-axial clamping can cause bending and twisting within the sample during the experiment. [10].

Considering these drawbacks, theoretical approaches were developed to calculate the shear properties of orthotropic materials. These approaches adopt the concept of isotropic materials, for which the shear modulus can be derived from the in-axis elastic modulus and the Poisson's ratio while the shear strength is derived from the in-axis strength. This kind of relation has been introduced and presented in Saint-Venant [11] and cited by Lekhnitskii [12]. Their approaches, however, are limited to the estimation of shear modulus and the accuracy of their results has never been investigated before. In this study, similar estimations for the shear properties are developed. The new approaches are based on the combination of both empirical and analytical in- and off-axis material properties, which enable them to be used to estimate both shear modulus and shear strength. The approaches are implemented for walnut wood (Juglans regia L.), for which a sufficient data set is available $[13,14]$. Furthermore, statistical analyses are performed to compare and validate the results with the shear properties that are experimentally obtained using the above-mentioned Arcan and shear block test setups. All six shear planes (Fig. 1), i.e., longitudinal radial (LR), radial longitudinal (RL), longitudinal tangential (LT), tangential longitudinal (TL) and radial tangential (RT), tangential radial (TR), of walnut wood are experimentally investigated at four different levels of moisture content.

This study also contributes to the completion of the orthotropic moisture-dependent data set of walnut wood. With the addition of the shear properties and in combination with the data from previous studies $[13,14]$, a comprehensive data set of walnut wood in the elastic and failure range is obtained. Walnut wood is valued for its wood being used for cabinets and furniture. Hence, a good part of historical objects is made of this wood. Therefore, this contribution on its properties may be valued for conservation purposes. 


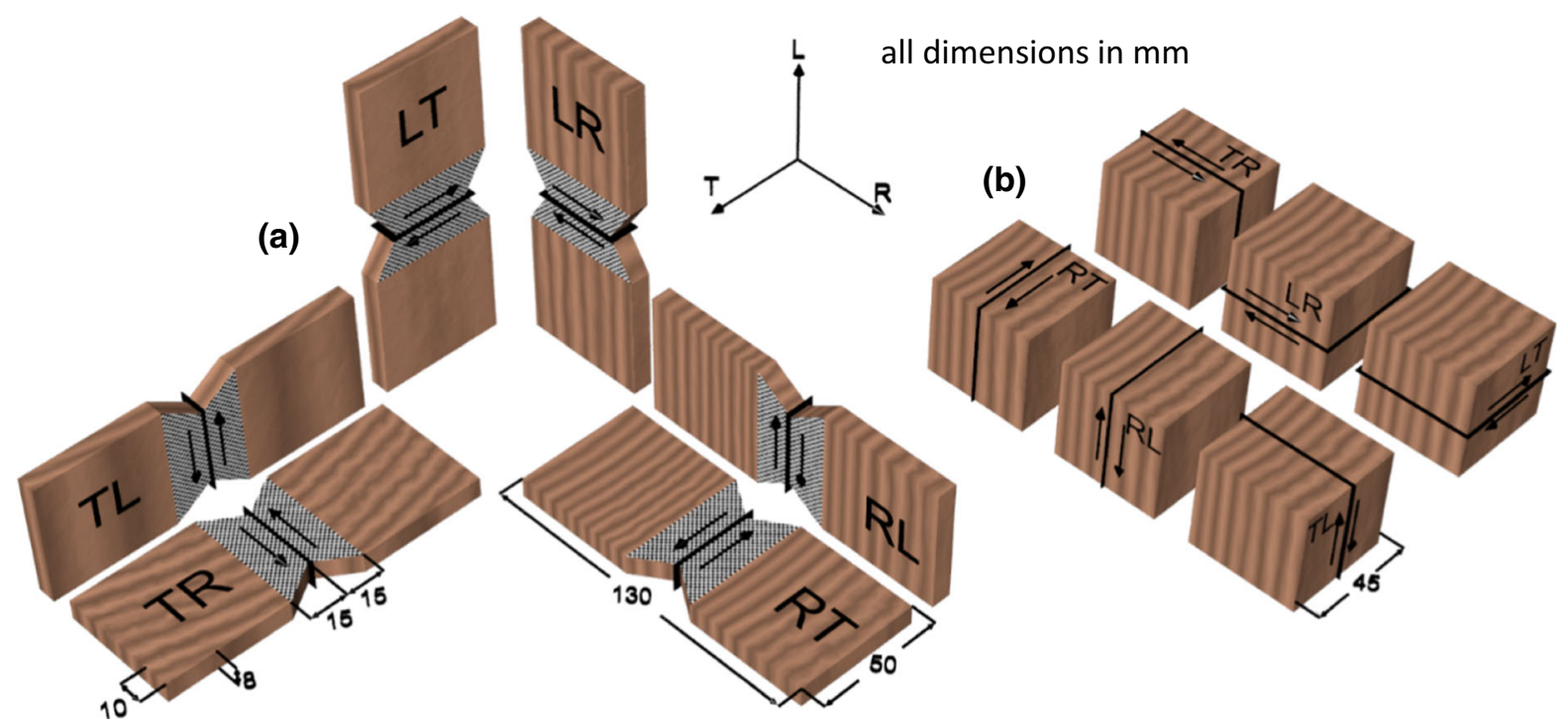

Fig. 1 Specimens for experimental test: a Arcan test, b shear block test

\section{Materials and methods}

\subsection{Material}

Walnut wood (Juglans regia L.) was taken from trees grown in the Caucasus region. At $11.8 \%$ moisture content, the average wood density of walnut wood is $671 \pm 34 \mathrm{~kg} / \mathrm{m}^{3}$. The samples used in this experiment were clear wood specimens without visible defects.

\subsection{Specimen preparation}

Blocks of wood with a dimension of at least $150 \times 100 \times 500 \mathrm{~mm}^{3}$ have been stored in four different moisture conditions ( $\mathrm{RH} \mathrm{50,65,85,95 \% )}$ at $20{ }^{\circ} \mathrm{C}$ for 1 month prior to the specimens preparation. Two different types of specimen were manufactured. The first type is the v-notched specimen used for the Arcan test. The specimens were originally cut to a wooden plate with a length of $130 \mathrm{~mm}$ and crosssection of $50 \times 8 \mathrm{~mm}^{2}$. Then, two notches were milled on the middle part of the specimen, which reduces the cross-section to $30 \times 8 \mathrm{~mm}^{2}$ (Fig. 1a). The second type is a wooden block with a dimension of $45 \times 45 \times 45 \mathrm{~mm}^{3}$. The specimen is introduced in DIN-52187 [7] for the shear strength test parallel to the fiber (Fig. 1b). After manufacturing, all specimens were returned to the climatic chamber for another month to ensure re-equilibrium of the specimens (mass variation less than $0.1 \%$ /day [15]).

\subsection{Density and moisture content measurements}

The density of the samples was measured during the process of sample preparation before the cutting of the notches. The densities were calculated as raw densities at specific moisture levels. The moisture content was determined after the experimental test by measuring at least fifteen random pieces of the tested specimens for each moisture condition. The measurement is based on the oven dry weight according to DIN-52183 [16] with the oven-dry mass method at $103{ }^{\circ} \mathrm{C}$ measured every $6 \mathrm{~h}$ until constant mass (change of mass less than $0.1 \%$ ) achieved ( $\pm 24 \mathrm{~h}$ drying).

\subsection{Shear test}

The shear moduli were determined by performing Arcan tests (Fig. 2a), which uses notched samples. The test is well-known due to investigations of various materials under shear loading (e.g., wood [6, 13], rocks [17], polymers [18] and composites [19, 20]). The loading schematic of the test is presented in Fig. 3. The shear strength was determined by the shear block test (Fig. 2b), which is based on DIN-52187 [7]. The loading schematic of shear block test is presented in Fig. 4. Both of these experimental tests were carried 
Fig. 2 Experimental test set-up: a Arcan test, b shear block test
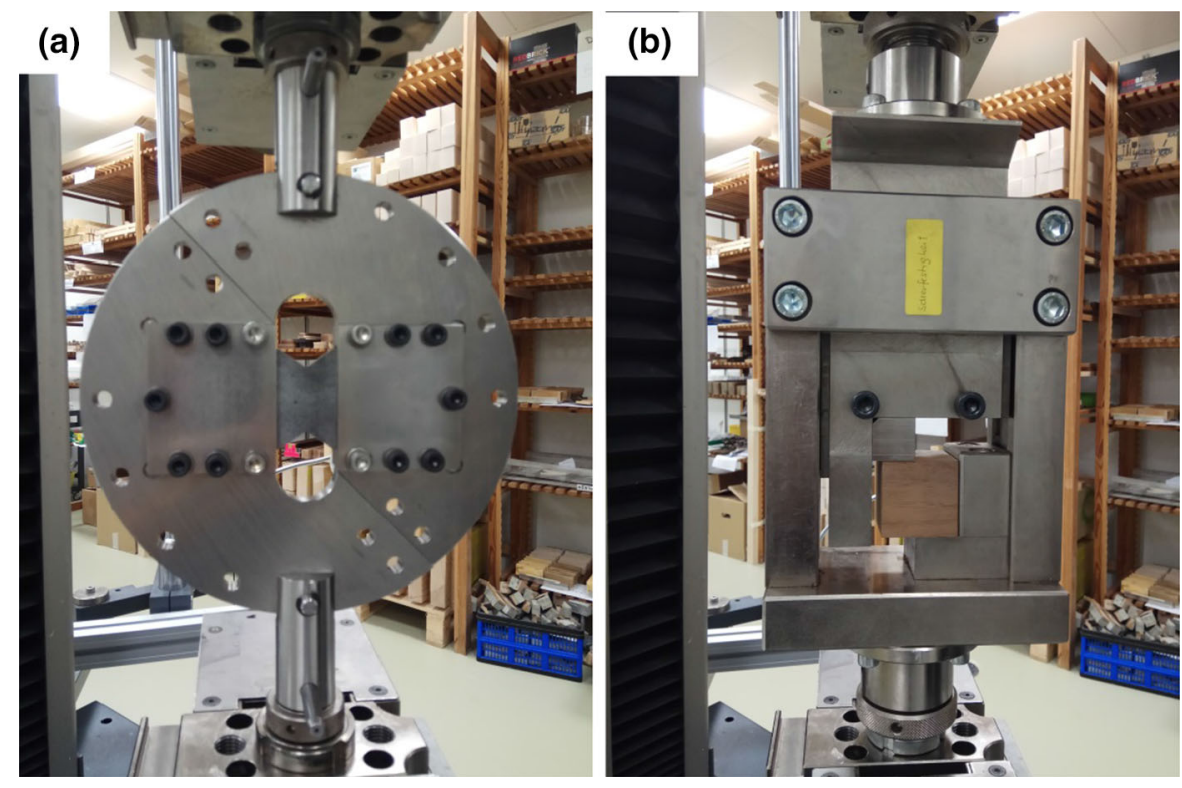

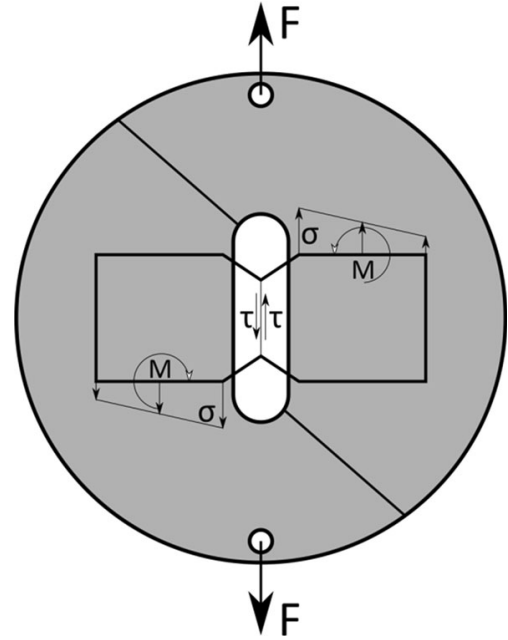

Fig. 3 Loading schematic for Arcan test. In the center of the specimen, which is the area of interest, stresses from tension $(\sigma)$ and moment (M) loadings are cancelled out and only the shear stresses $(\tau)$ are remaining

out on a Universal Testing Machine (Zwick Roell Z100, Zwick Roell, Ulm, Germany) equipped with a $100 \mathrm{kN}$ load cell.

The Arcan test was conducted on all six shear planes of wood (LR, RL, LT, TL, RT and TR) (Fig. 1a). At least seven successful tests on each plane were obtained. An initial force of $10 \mathrm{~N}$ was set as the starting point of the measurements. The tests were run in a displacement-controlled mode with a rate of
$1 \mathrm{~mm} / \mathrm{min}$ until failure. For the strain measurement, digital image correlation (DIC) was used. A high contrast speckle pattern was applied to the middle area on both sides of the specimens (Fig. 1a) with an airbrush gun in combination with a nozzle cup with a diameter of $0.8 \mathrm{~mm}$ and an airbrush needle with a diameter of $0.4 \mathrm{~mm}$. During the measurement, pictures with a frequency of $1 \mathrm{~Hz}$ were taken using two cameras. Each camera was aiming straight on each side of the specimens taking images of the speckled regions. Based on these sets of images, the surface strains were calculated with a commercial twodimensional digital image correlation software (VIC 2D, Correlated Solution). This technique has been successfully used in previous studies [13, 21, 22]. The shear moduli $(G)$ were calculated by a linear relation between shear stress and shear strain between 20 and $40 \%$ of the maximum shear stress.

The shear block test was also performed on all six shear planes of wood (Fig. 1b) on the same testing machine and load cell. Ten successful tests on each plane were obtained. An initial force of $500 \mathrm{~N}$ was set as the starting point continued by a displacement controlled mode with a rate of $4 \mathrm{~mm} / \mathrm{min}$ until failure. In this measurement, the focus was set on measuring the shear strength. Therefore, the strain was just calculated from the displacement of the machine lever. The shear strength $\left(\tau_{\mathrm{u}}\right)$ was determined as the ultimate stress followed by failure. 

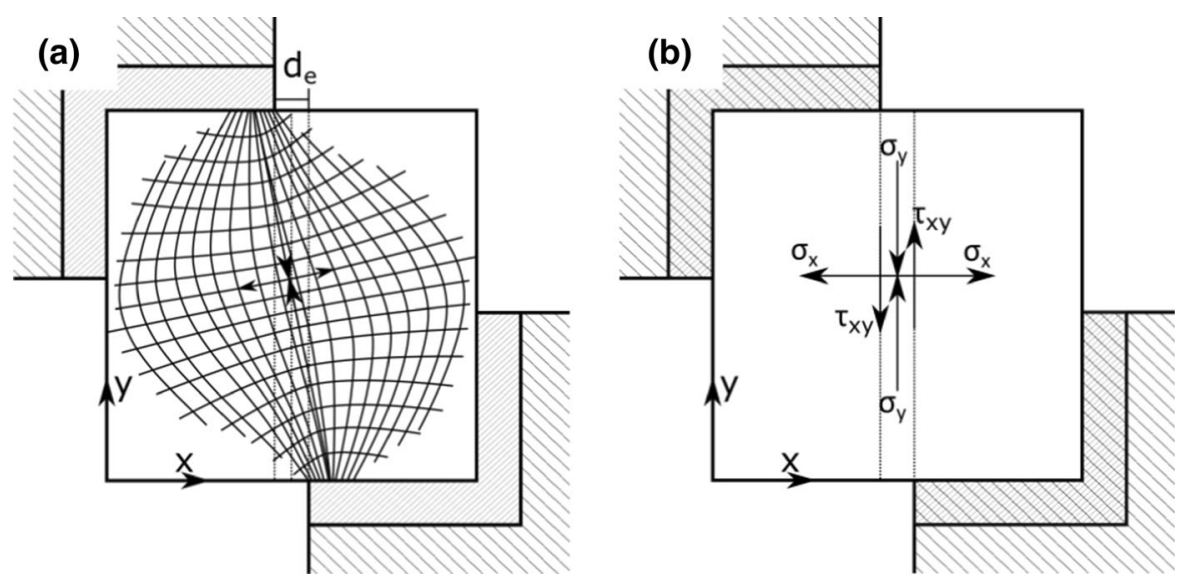

Fig. 4 Shear distribution in loaded shear block specimens: a off-axis stresses due to eccentricity $\left(d_{e}\right)$ [36], b orthogonal stress components

\subsection{Indexing of the shear properties}

The assigning of the six shear planes with the indices $L, R, T$ is defined in several different ways in literature, which can lead to confusion regarding the shear moduli $(G)$ and shear strength $\left(\tau_{\mathrm{u}}\right)$ of the different planes. In this study, the following assignment is consistently applied to all shear properties: Each shear plane is assigned with two indices. The first index refers to the direction normal to the shear plane, while the second index refers to the loading direction. As an example, $G_{\mathrm{RL}}$ and $\tau_{\mathrm{uRL}}$ are properties acting on the surface normal to the radial $(R)$ direction while being loaded in longitudinal $(L)$ direction. This indexing is consistent with the shear stresses ( $\tau$ in Fig. 5). The specimens as presented in Fig. 1 have also been named regarding these notations.

\subsection{Numerical approaches}

The numerical approaches introduced in this study can be used to estimate both shear modulus and shear strength. All methods to estimate the shear moduli $(G)$ require the elastic moduli $\left(E_{\mathrm{L}}, E_{\mathrm{R}}, E_{\mathrm{T}}\right)$ and the Poisson's ratios $\left(v_{\mathrm{LR}}, v_{\mathrm{RL}}, v_{\mathrm{LT}}, v_{\mathrm{TL}}, v_{\mathrm{RT}}, v_{\mathrm{TR}}\right)$ as the input parameter. On the other hand, the input parameter to estimate the shear strengths $\left(\tau_{\mathrm{u}}\right)$ are the in-axis strengths $\left(\sigma_{\mathrm{uL}}, \sigma_{\mathrm{uR}}, \sigma_{\mathrm{uT}}\right)$. All input parameters for walnut wood (Juglans regia $\mathrm{L}$.) based on $[13,14]$ are presented in Table S1.

The numerical approaches are adopting the advantage of Hankinson's empirical formula [23], which is

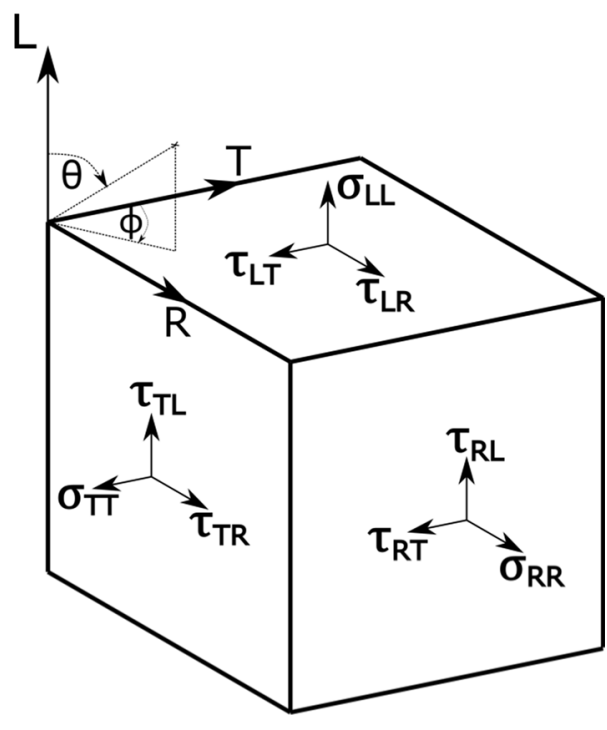

Fig. 5 Stress block and spherical coordinate system

able to estimate the off-axis parameter with only the knowledge of the in-axis properties without the shear properties. Although this equation was initially developed to predict the off-axis strength of a material $[8,23]$, the formula has also been used to estimate the off-axis elastic moduli with sufficient accuracy [24, 25]. Hankinson's formulas in three-dimensional form, which differentiate between the relation within the perpendicular planes (LR-, LT-plane) (Eq. (1)) and the parallel plane (RT-plane) (Eq. (2)), are used in this study. 
$N_{(\phi)}=\frac{N_{\mathrm{R}}-N_{\mathrm{T}}}{\pi / 2} \phi+N_{\mathrm{T}}-\frac{1}{2} K\left(N_{\mathrm{R}}+N_{\mathrm{T}}\right) \sin (2 \phi)$

$N_{(\theta, \phi)}=\frac{N_{\mathrm{L}} N_{(\phi)}}{N_{\mathrm{L}} \sin ^{2} \theta+N_{(\phi)} \cos ^{2} \theta}$

with $N_{(\phi)}$ being the empirical value of the off-axis parameter ( $N$ stands for either elastic moduli, Poisson's ratio, or strength) in the perpendicular plane with an angle $\phi$, which defines the offset in the RT-plane ( $\phi=0$ is the tangential direction $T, \phi=\pi / 2$ is the radial direction $R) . N_{(\theta, \phi)}$ is the off-axis parameter with the angle $\theta$ in respect to fiber direction $(\theta=0$ is in fiber direction $(L), \theta=\pi / 2$ is perpendicular to fiber direction). Both $\theta$ and $\phi$ are angles for a spherical coordinate system as presented in Fig. 5. $K$ is an empirically determined constant to adjust the amplitude of the sinusoidal function for RT-plane $(K=0.2$ for hardwood, $K=0.4$ for softwood [8]).

\subsubsection{Empirical and analytical approaches for estimating shear moduli}

\section{Approach M1 (Modulus 1)}

Approach M1 is based on the combination of Hankinson's formula, which estimates the off-axis parameter for elastic modulus, and Eq. (3), which relates the off-axis elastic moduli $\left(E_{\theta \mathrm{ij}}\right)$ to the shear modulus $(G)$ by tensor rotation [26, 27]. The results of substituting both equations together are the definition of the shear modulus $(G)$ as presented in Eqs. (4) and (5)

$E_{\theta_{i j}}=\left[\frac{\cos ^{4} \theta}{E_{i}}+\frac{\sin ^{4} \theta}{E_{j}}+\left(\frac{1}{G_{i j}}-\frac{v_{j i}}{E_{i}}-\frac{v_{i j}}{E_{j}}\right) \cos ^{2} \theta \sin ^{2} \theta\right]^{-1}$,

where, $i, j \in \mathrm{L}, \mathrm{R}, \mathrm{T}$ and $i \neq j$,

$G_{i j}=\left[\frac{v_{j i}+1}{E_{i}}+\frac{v_{i j}+1}{E_{j}}\right]^{-1}$,

where, $i, j=\mathrm{L}, \mathrm{R}$, or $\mathrm{L}, \mathrm{T}$,

$G_{i j}=\left[\frac{v_{j i}-1}{E_{i}}+\frac{v_{i j}-1}{E_{j}}+\frac{8}{(1-K)\left(E_{i}+E_{j}\right)}\right]^{-1}$,

where, $i, j=\mathrm{R}, \mathrm{T}$, with $G_{i j}$ being the shear modulus in $i j$-plane. $E_{i}$ and $E_{j}$ are the elastic moduli along $i$ - and $j$-axis, respectively. $v_{i j}$ is the Poisson's ratio that corresponds to a passive contraction in direction $i$ when an extension is applied in direction $j$ and vice versa for $v_{j i}$.

\section{Approach M2 (Modulus 2)}

The shear modulus is estimated using the same equation as for the isotropic material but with the elastic modulus and Poisson's ratio of the $45^{\circ}$ off-axis angle (Eq. (6))

$G_{i j}=\frac{E_{45_{i j}}}{2\left(1+v_{45_{i j}}\right)}, \quad$ where, $i, j \in \mathrm{L}, \mathrm{R}, \mathrm{T}$

and $i \neq j$.

This relation was initially introduced in Kon [28] and cited in Yoshihara [24]. It can also be obtained by solving the combination of Hankinson's formula (Eqs. (1) and (2)), the off-axis tensor rotation of elastic modulus (Eq. (3)) and the Poisson's ratio [27] for $\theta=45^{\circ}$. In this study, both $45^{\circ}$ off-axis elastic modulus $\left(E_{45 i j}\right)$ and Poisson's ratio $\left(v_{45 i j}\right)$ are estimated based on Hankinson's formula. Therefore, it leads to Eqs. (7) and (8)

$G_{i j}=\left[\left(1+\frac{2}{\frac{1}{v_{i j}}+\frac{1}{v_{j i}}}\right)\left(\frac{1}{E_{i}}+\frac{1}{E_{j}}\right)\right]^{-1}$,

where, $i, j=\mathrm{L}, \mathrm{R}$, or $\mathrm{L}, \mathrm{T}$,

$G_{i j}=\frac{(K-1)\left(E_{i}+E_{j}\right)}{2(K-1)\left(v_{i j}+v_{j i}\right)-4}, \quad$ where, $i, j=\mathrm{R}, \mathrm{T}$.

Hankinson's formula has never been used to estimate the off-axis Poisson's ratio. However, since it is known that the difference between approach M1 and M2 is only on their way to assume the Poisson's ratio, the difference in their resulted shear modulus will directly describe the agreement between their assumptions.

Two other approaches (Eqs. (9) and (10)) for estimating the shear moduli in all six shear planes have been introduced in Saint-Venant [11] and cited in Lekhnitskii [12]. 
Approach M3 (Modulus 3)

$G_{i j}=\left[\frac{v_{j i}+1}{E_{i}}+\frac{v_{i j}+1}{E_{j}}\right]^{-1}, \quad$ where, $i, j \in \mathrm{L}, \mathrm{R}, \mathrm{T}$ and $i \neq j$.

\section{Approach M4 (Modulus 4)}

$G_{i j}=\left[\frac{v_{j i}}{E_{i}}+\frac{v_{i j}}{E_{j}}+\frac{2}{\sqrt{E_{i} E_{j}}}\right]^{-1}$,

where, $i, j \in \mathrm{L}, \mathrm{R}, \mathrm{T}$ and $i \neq j$.

It is noticed that the first approach introduced by Saint-Venant [11] (M3) (Eq. (9)) is identical to the M1 approach, based on Hankinson's formula for LR- and LT-plane (Eq. (4)). The M3 approach, therefore, can be seen as a simplification method of the M1, since it can be regarded as a combination of two-dimensional Hankinson's formula in the 2D version and the tensor rotation. Therefore, unlike M1, which requires two different equations (Eqs. (4) and (5)), the M3 approach only requires one equation for all the three shear planes (Eq. (9)). However, this similarity seems only a coincidence when it is tracked back to the beginning when both Hankinson's and M3 approach were introduced. St. Venant initially proposed M3 relation for shear modulus in 1863, while Hankinson's formula, which was developed later in 1921, is initially aiming for the material strength. Only later in [24, 25], Hankinson's formulas were used to estimate the offaxis elastic moduli. In this study, both approaches are used for estimating the shear modulus. The formulas are identical for the LT- and LR-plane, yet differ for the RT-plane (Eqs. (5) and (9)).

\subsection{Estimation of shear strength}

To estimate shear strength, alternative approaches to calculate the off-axis strength properties are combined with Hankinson's formula. However, unlike the shear moduli, which can be analytically derived with tensor rotation as in M1 (Eq. (3)), the approaches for the shear strength are derived from failure criteria taken from literature.

\section{Approach S1 (Strength 1)}

Here, the off-axis strength is estimated based on the ellipsoidal failure criterion [29]

$$
\sigma_{u \theta i j}^{2}=\left[\frac{\cos ^{4} \theta}{\sigma_{u_{i}}^{2}}+\frac{\sin ^{4} \theta}{\sigma_{u_{j}}^{2}}+\left(\frac{1}{\tau_{u_{i j}}}\right) \cos ^{2} \theta \sin ^{2} \theta\right]^{-1},
$$

where, $i, j \in \mathrm{L}, \mathrm{R}, \mathrm{T}$ and $i \neq j$,

with $\tau_{u i j}$ being the shear strength in $i j$-plane. $\sigma_{u \theta i j}$ are the off-axis strength properties at angle $\theta . \sigma_{u i}$ and $\sigma_{u j}$ are the strength properties in $i$ and $j$ axis, respectively. The shear strengths (Eqs. (12) and (13)) can be obtained by substituting the off-axis strength properties of Hankinson's formula (Eqs. (1) and (2)) to Eq. (11)

$\tau_{u_{i j}}=\frac{1}{2}\left[2 \sigma_{u_{i}} \sigma_{u_{j}}\right]^{0.5}, \quad$ where, $i, j=\mathrm{L}, \mathrm{R}$, or $\mathrm{L}, \mathrm{T}$,

$\tau_{u_{i j}}=\left[\frac{16}{(K-1)^{2}\left(\sigma_{u_{i}}+\sigma_{u_{j}}\right)^{2}}-\frac{1}{\sigma_{u_{i}}^{2}}-\frac{1}{\sigma_{u_{j}}^{2}}\right]^{-0.5}$,

where, $i, j=\mathrm{R}, \mathrm{T}$.

\section{Approach S2 (Strength 2)}

This approach takes the off-axis strength form the failure criterion introduced by Norris [30]

$$
\sigma_{u \theta i j}^{2}=\left[\frac{\cos ^{4} \theta}{\sigma_{u_{i}}^{2}}+\frac{\sin ^{4} \theta}{\sigma_{u_{j}}^{2}}+\left(\frac{1}{\tau_{i j}^{2}}-\frac{1}{\sigma_{u_{i}} \sigma_{u_{j}}}\right) \cos ^{2} \theta \sin ^{2} \theta\right]^{-1},
$$$$
\text { where, } i, j \in \mathrm{L}, \mathrm{R}, \mathrm{T} \text { and } i \neq j \text {. }
$$

The shear strengths are obtained by substituting offaxis strength properties obtained with Hankinson's formula (Eqs. (1) and (2)) to Eq. (14). The results are presented in Eqs. (15) and (16)

$\tau_{u_{i j}}=\frac{1}{3}\left[3 \sigma_{u_{i}} \sigma_{u_{j}}\right]^{0.5}, \quad$ where, $i, j=L, R$, or $L, T$,

$\tau_{u_{i j}}=\left[\frac{16}{(K-1)^{2}\left(\sigma_{u_{i}}+\sigma_{u_{j}}\right)^{2}}+\frac{1}{\sigma_{u_{i}} \sigma_{u_{j}}}-\frac{1}{\sigma_{u_{i}}^{2}}-\frac{1}{\sigma_{u_{j}}^{2}}\right]^{-0.5}$, where, $i, j=\mathrm{R}, \mathrm{T}$.

\section{Approach S3 (Strength 3)}

The off-axis strength is estimated based on a failure criterion introduced by [31] 
$\sigma_{u \theta i j}^{2}=\left[\frac{\cos ^{4} \theta}{\sigma_{u_{i}}^{2}}+\frac{\sin ^{4} \theta}{\sigma_{u_{j}}^{2}}+\left(\frac{1}{\tau_{i j}^{2}}-\frac{1}{\sigma_{u_{i}}^{2}}-\frac{1}{\sigma_{u_{j}}^{2}}+\frac{1}{\sigma_{u_{k}}^{2}}\right) \cos ^{2} \theta \sin ^{2} \theta\right]^{-1}$,

where, $i, j, k \in \mathrm{L}, \mathrm{R}, \mathrm{T}$ and $i \neq j \neq k$.

In Tsai, $\mathrm{Wu}$ [32], the Hill failure criterion was simplified with an assumption that the strengths in the perpendicular directions are equal $\left(\sigma_{\mathrm{uR}}=\sigma_{\mathrm{uT}}\right)$ while the parallel plane is observed (LR- and LT-planes). This is known as the Tsai-Hill failure criterion. By adopting this assumption and substituting Hankinson's formula to Eq. (17), the shear strength can be estimated as in Eqs. (18) and (19). The assumption also ensures that the square root result of Eq. (18) never leads to an imaginary number

$\tau_{u_{i j}}=\left[\left(\frac{1}{\sigma_{u_{i}}}+\frac{1}{\sigma_{u_{j}}}\right)^{2}-\frac{1}{\sigma_{u_{k}}^{2}}\right]^{-0.5}$,

where, $i, j, k=\mathrm{L}, \mathrm{R}, \mathrm{R}$ or $\mathrm{L}, \mathrm{T}, \mathrm{T}$,

$\tau_{u_{i j}}=\left[\frac{16}{(K-1)^{2}\left(\sigma_{u_{i}}+\sigma_{u_{j}}\right)^{2}}-\frac{1}{\sigma_{u_{k}}^{2}}\right]^{-0.5}$,

where, $i, j, k=\mathrm{R}, \mathrm{T}, \mathrm{L}$.

\subsection{Statistical analysis}

The material properties in main orthogonal directions from the literature [13] were determined by experimental tests. Each property was the averaged value from several tested specimens. Therefore, it contains a certain amount of deviation. Since the shear moduli and shear strengths are estimated from these data, their variation is taken into account by the bootstrapping technique with one random sample for each parameter and repetition of 20,000 times. As an additional check, the results have been compared and show agreement with the error propagation calculation based on the combined standard deviation for each formula [33].

To compare the calculated shear data and the experimentally determined data, a statistical analysis was performed via $T$ test (significance level 0.05). The statistical analysis provides a reliable comparison regarding the differences between these data. The results of the statistical comparison between the calculated shear data and the experimental data are either no significant difference (data come from the same population) or significant difference (data come from the different population). The error propagation was also considered in the analysis. The abovementioned error propagation methods testify the variation of data in the form of standard deviation for each material property calculated with the theoretical approaches. Based on this standard deviation, a set of normally distributed random numbers is generated for the calculated shear data with an equal quantity to the experimentally tested specimens. The comparison method is repeated 1000 times and the results are taken based on the averaged probability value. Prior to this $T$ test analysis, tests are conducted
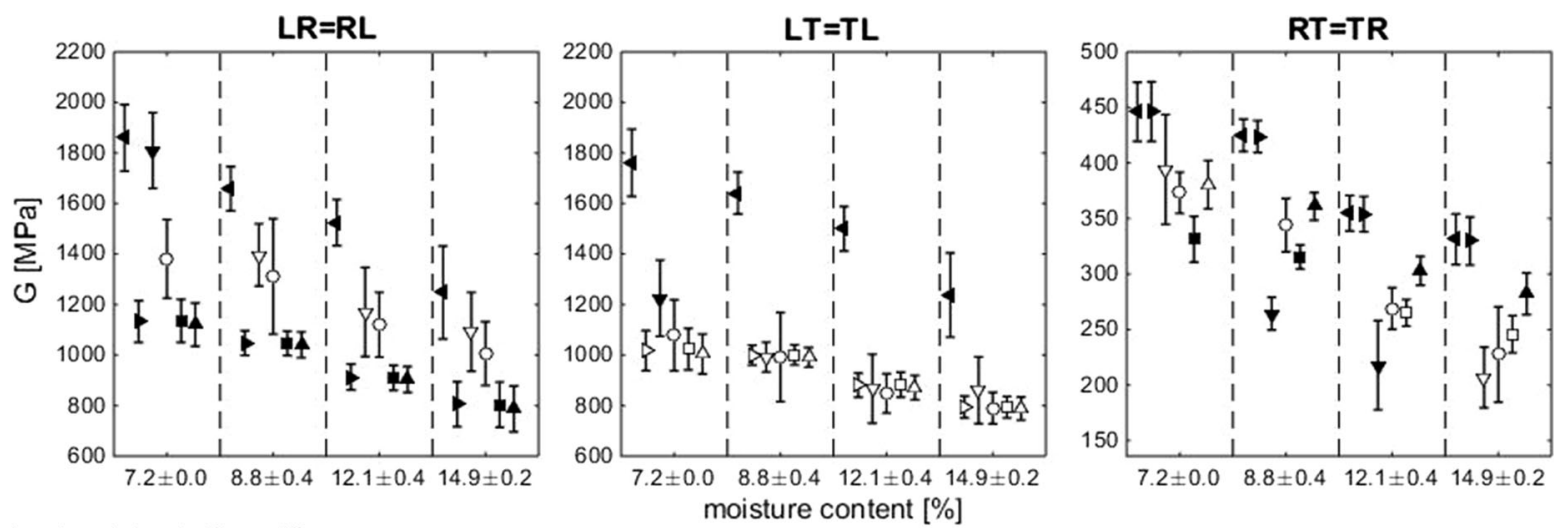

closed symbol $=$ significant difference opened symbol $=$ no significant difference

$\neg-R \rightarrow U \longrightarrow \mathrm{R} \longrightarrow \mathrm{M} 1 \multimap-\mathrm{M} 2 \multimap \mathrm{M} 3 \multimap \mathrm{M} 4$

Fig. 6 Shear moduli of walnut wood (Juglans regia L.) determined by various methods: Reference data (R) (mechanical Arcan test), Ultrasound data (U) [13], and the different theoretical approaches (M1-M4), which are described in the text. The closed or opened symbols indicate statistically significant and no significant difference to the reference data, respectively. The dashed line separates the data of the different moisture levels; the $\mathrm{x}$-axis is not scaled 
on the normal distribution of the data via Kolmogorov-Smirnov's test [34] and on the variance equality of each data pair via Bartlett's test [35].

\section{Results and discussion}

\subsection{Experimentally determined shear moduli}

The experimentally determined shear moduli $(G)$ of walnut wood are presented in Fig. 6 and in Table S2 and S3 (supplementary online information) together with the different theoretically calculated values. At $11.8 \%$ moisture content, the shear moduli of walnut wood are $1115( \pm 15 \%), 849( \pm 12 \%)$ and $270( \pm 9 \%)$ MPa in LR-, LT- and RT-planes, respectively. Two previous studies report shear moduli of walnut wood $[13,26]$, which are lower by $2-28 \%$ than those of the present study. Even within the same wood species, the natural inhomogeneity, different sources, different density and different tested moisture content of wood may lead to the variation of wood properties $[8,36]$. The difference in the properties of the wood is even higher when comparing different wood species due to differences in tissue and cell wall structure [8]. The shear moduli of maritime pine (Pinus pinaster Ait.) and common ash (Fraxinus excelsior L.), which are tested in Xavier et al. [6] and Clauss et al. [21], respectively, are up to 28 and $45 \%$ higher in comparison to our results.
An alternative method to experimentally estimate the shear moduli is the ultrasound test. This technique and its theoretical basis have been very well established [37]. For walnut wood, Bachtiar et al. [13] have used the ultrasound technique to estimate its moisture dependent shear properties. The results, which are presented in Fig. 6 and also in Table S3, are statistically compared with the data obtained by the Arcan test. It is shown that the results of the ultrasound tests are in reasonable agreement with those of the Arcan tests. For the LR-and LT-plane, the results of three out of four tested moisture levels show no significant difference compared to those of the Arcan tests, while in RT-plane, two out of four show no significant difference. The slight differences are suspected to be due to the natural variability of wood properties, as different specimens have been tested for the two studies.

\subsection{Experimentally determined shear strength}

The shear strengths $\left(\tau_{\mathrm{u}}\right)$ of walnut wood were investigated with the shear block test. The results are presented in Fig. 7 and in Table S4 and S5 (supplementary online information). When loading parallel to fiber direction (RL- and TL-planes), the shear block test is recognized as a standard norm to investigate the shear strengths [7]. By inducing loading in the shear plane across the fiber (LR and LT in Fig. 8), mainly compression failure will occur. Since wood is

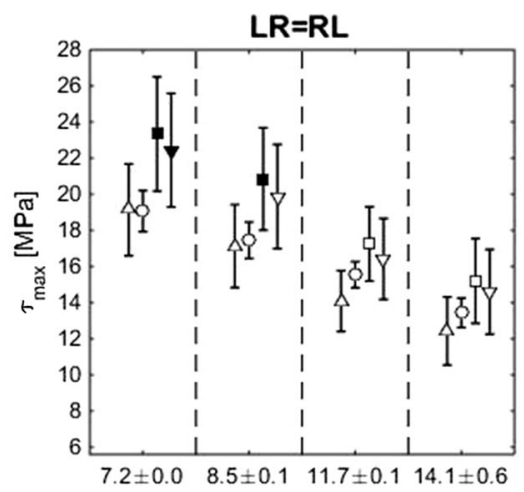

closed symbol $=$ significant difference opened symbol $=$ no significant difference
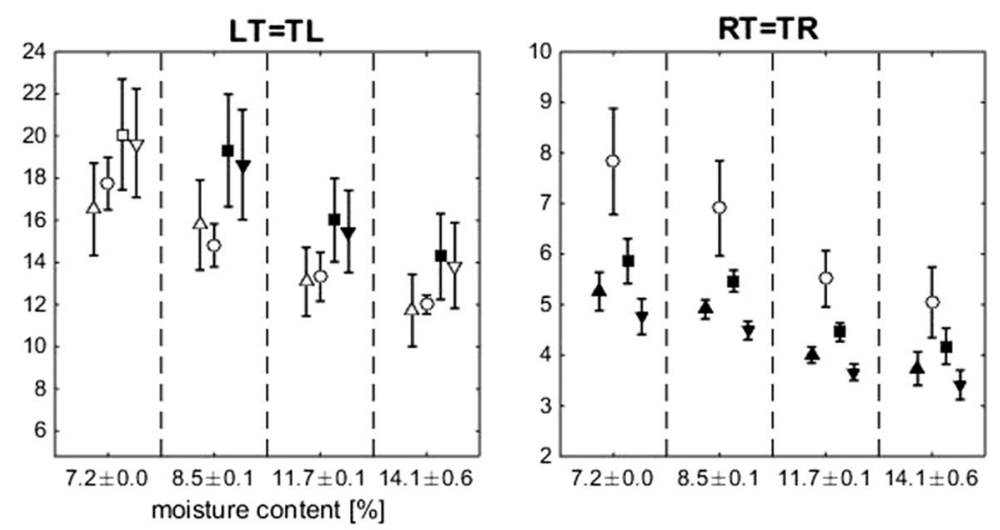

$\longrightarrow \mathrm{O}-\mathrm{R}-\mathrm{S} 1 \rightarrow \mathrm{S} 2 \rightarrow \mathrm{S} 3$
Fig. 7 Shear strength $\left(\tau_{\mathrm{u}}\right)$ of walnut wood (Juglans regia L.) determined by various methods: Reference data (R) (mechanical shear block test) and the different theoretical approaches (S1S3) (see text for details and equations). The closed or opened symbols indicate statistically significant and no significant difference to the reference data, respectively. The dashed line separates the data of the different moisture levels; the $\mathrm{x}$ axis is not scaled 
(a)
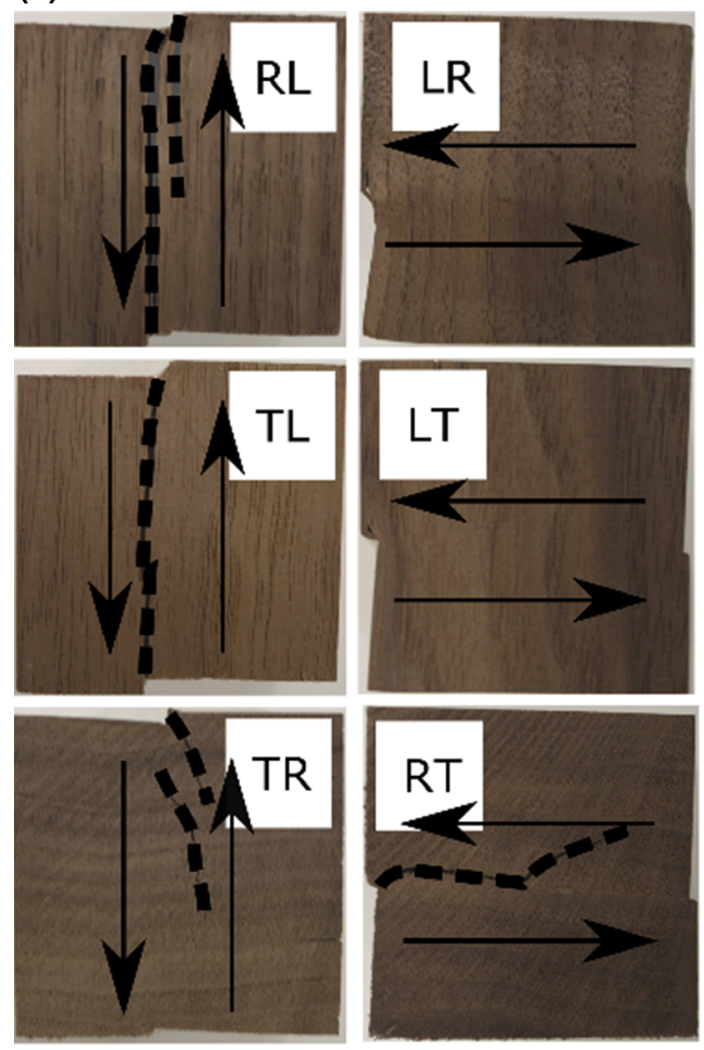

(b)
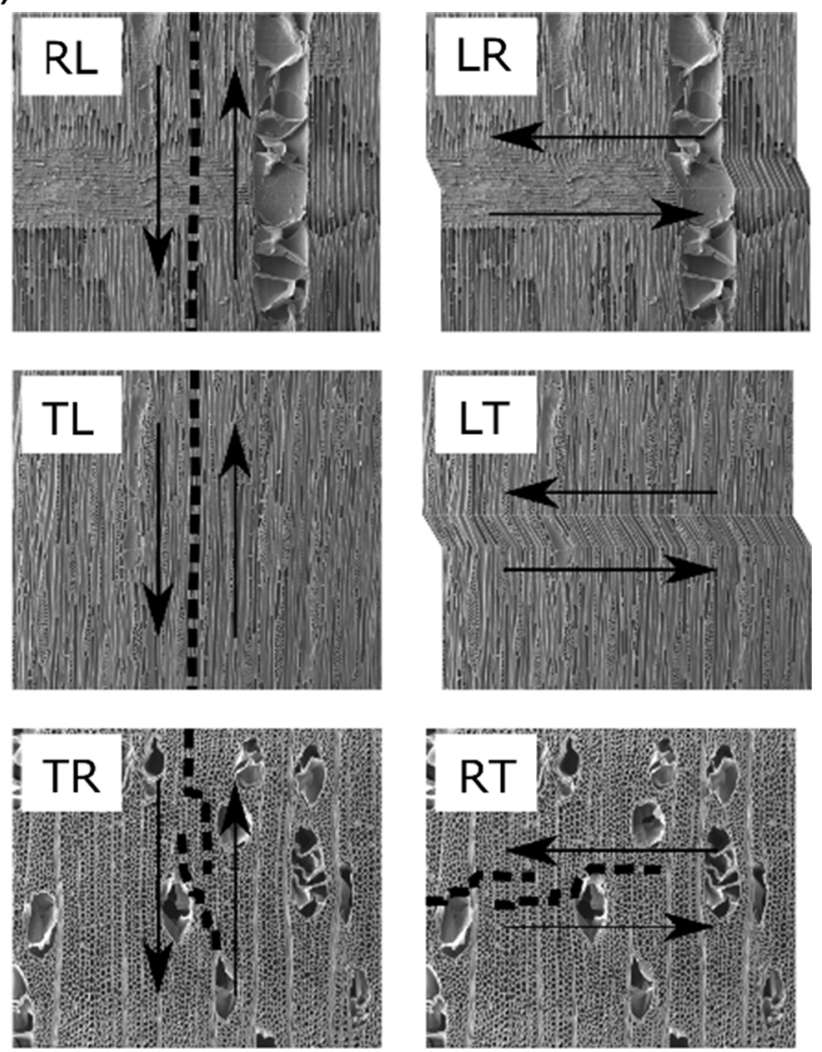

Fig. 8 Shear failure pattern of walnut wood (Juglans regia L.): a shear block test. b Cellular level (assumed failure pattern based on the pattern of the shear block test). Arrows indicate the

modelled as an orthotropic material, which assumes a symmetry of its properties, the resulting shear strengths in RL- and TL-planes are also regarded to represent the shear strengths LR- and LT-planes $\left(\tau_{\mathrm{uLR}}=\tau_{\mathrm{uRL}}\right.$ and $\left.\tau_{\mathrm{uLT}}=\tau_{\mathrm{uTL}}\right)$.

Determination of shear strength in the RT-plane has never been defined in the standard norm, which may be due to the problem of failing not purely in shear, and the norms being more focussed on application aspects. However, from a scientific point of view, the shear strength in RT-plane is required for a full threedimensional material model. Therefore, in this study, the shear strength in RT-plane is also estimated with the shear block test. The specimens were tested in both RT- and TR-planes and the results were averaged. The oblique crack pattern (TR and RT) indeed indicates a non-pure shear failure. However, the results are still regarded as the optimum for representing shear strength [38]. applied shear loading while dashed lines indicate the eventually appearing cracks during failure

At $11.8 \%$ moisture content, the shear strengths of walnut wood obtained in this study are $15.5( \pm 5 \%)$ $\mathrm{MPa}, 13.3( \pm 9 \%) \mathrm{MPa}$ and $6.2( \pm 7 \%) \mathrm{MPa}$ in LR-, LT- and RT-planes, respectively. The shear strengths of walnut wood as reported in literature [39] are lower by almost a factor of two compared to the now obtained results. The literature data in LR- and LTplane are compiled from walnut (Juglans regia L.) and black walnut (Juglans nigra L.) wood, while in RTplane, the data was estimated from oak wood (Quercus robur L.) and beech wood (Fagus sylvatica L.). These two aspects make a direct comparison to our results rather difficult.

On the other hand, the comparison of the presently obtained data with that of other wood species show similar shear strengths in the LR- and LT-planes. Two hardwood species, oak (Quercus robur L.) and Sycamore maple (Acer pseudoplatanus L.) wood were tested using the shear block test and presented in 
Ozyhar et al. [40] and Sonderegger et al. [41], respectively. The values are higher by $3-15 \%$ compared to the ones of the current study, which is well within the range of natural variability of properties, especially considering different wood species. Moreover, the shear strength in RT-plane of maritime pine wood (Pinus pinaster Ait.) [6], as determined by the Arcan and the Iosipescu test was 25-29\% lower than the presently obtained one of walnut wood. This is in line with the general observation that most softwood species possess a lower shear strength than hardwood species [8,39].

\subsection{Influence of fiber direction on shear modulus and strength}

As shown in Figs. 6 and 7 and discussed in the previous sections, shear modulus and strength of wood are highest in RL $=$ LR-plane, followed by TL $=$ LTplane, whereas the $\mathrm{RT}=\mathrm{TR}$-plane represents the weakest one. This order of stiffness and strength is related to the in-axis material properties of wood $(\mathrm{L}>\mathrm{R}>\mathrm{T})$. The reason for this specific order lies in the orthotropic cellular structure of the wood as presented in Fig. 8b.

Both, shear modulus and shear strength in TL-plane are always lower than those in RL-plane. This may be explained by the fact that the TL-shear plane is associated with the deformation in L-direction, which is in plane with the wood rays. In this shear plane, the middle lamellas are also aligned continuously, which may lead to a smooth plane of intra-wall deformations and failures between adjacent fibers or between fibers and rays [42]. This type of failure requires less energy in comparison to that associated with the RL-plane, where primarily trans-wall deformations or failure (exposing the cell lumens) occur. On the other hand, RT- and TR- shear plane always possess the lowest resistance against shear. Loading in these shear planes leads to deformation in plane with the wood fibers.

Based on the theoretical assumption of the orthotropic material, the shear moduli and strengths show symmetric behaviour: $G_{\mathrm{LT}}=G_{\mathrm{TL}}, G_{\mathrm{LR}}=G_{\mathrm{RL}}$, $G_{\mathrm{RT}}=G_{\mathrm{TR}}, \tau_{\mathrm{uLT}}=\tau_{\mathrm{uTL}}, \tau_{\mathrm{uLR}}=\tau_{\mathrm{uRL}}$ and $\tau_{\mathrm{uRT}}=-$ $\tau_{\mathrm{uTR}}$ [8]. While this assumption is understandable for the shear moduli, its interpretation for the shear strength is rather difficult. The RL and LR shear strengths $\left(\tau_{\mathrm{uRL}}\right.$ and $\left.\tau_{\mathrm{uLR}}\right)$ are assumed to be equal, as well as the TL and LT shear strength $\left(\tau_{\mathrm{uTL}}\right.$ and $\left.\tau_{\mathrm{uLT}}\right)$ are. Yet, the specific failure patterns of the respective planes are completely different. Shear loading parallel to fiber direction $\left(\tau_{\mathrm{uRL}}\right.$ and $\left.\tau_{\mathrm{uTL}}\right)$ induces both transand intra-wall failure. On the other hand, shear loading perpendicular to fiber direction $\left(\tau_{\mathrm{uLR}}\right.$ and $\left.\tau_{\mathrm{uLT}}\right)$ always leads to kinking of the wood fibers. The high resistance of wood fibers against kinking leads to compressive failure instead.

\subsection{Influence of moisture content on shear modulus and strength}

The shear moduli and shear strengths decrease for increasing moisture content. The shear moduli in LRplane $\left(G_{\mathrm{LR}}\right)$ decrease from $1315( \pm 4 \%) \mathrm{MPa}$ to $972( \pm 4 \%) \mathrm{MPa}$ as the moisture content increases from 7.2 to $14.7 \%$. This behaviour is well-known from previous studies (e.g. Bachtiar et al. [13]). In general, wood mechanical properties including the shear moduli and strengths decrease as the moisture content increases [36, 43]. This is due to the reduction of the number of hydrogen bonds crosslinking the cellulose and other constituents in the non-crystalline regions of the cell walls as moisture content increases [44, 45]. Part of this effect is also due to the increase of the cross-sectional area due to swelling without adding material other than water [36] which leads to a lower calculated material properties.

\subsection{Theoretical approaches for estimating the shear modulus}

The numerically derived values for shear moduli obtained from the different theoretical approaches and their significance level in comparison to the mechanically estimated data are presented in Fig. 6 and in Table S3. The best estimations are given by the M1 approach with average deviations of $-19,0$ and $-3 \%$ in comparison to the Arcan data for $G_{L R}, G_{L T}$ and $\mathrm{G}_{\mathrm{RT}}$, respectively.

In both LR- and LT-planes, the M1 approach uses the same equation to estimate the shear moduli (Eq. (4)). Interestingly, the comparison between the shear moduli calculated with this approach and those obtained by the Arcan tests shows an underestimation of the shear moduli in LR-plane $\left(G_{\mathrm{LR}}\right)$ by around $19 \%$. In LT-plane, however, the differences are below the level of significance at all moisture levels. Since the M1 approach is based on the combination of 
Hankinson's empirical formula and the analytically derived tensor rotation, one may conclude that the inaccuracy in the LR-plane originates from Hankinson's formula, which might fail to correctly estimate the off-axis material parameters in this plane. However, Yoshihara [24] has investigated the off-axis parameter of two wood species, agathis (Agathis sp.) and katsura (Cercidiphyllum japonicum Sieb. et Zucc.) wood in LR-plane. The results show a good agreement between the experimental data, the Hankinson data and the tensor rotation data for both wood species. The inaccuracy visible for the LR-plane in this study is likely due to the variability of the wood. Different sets of wood specimens were taken for the Arcan test and the in-axis test [13]. Therefore, a variation of density or other parameters cannot be avoided.

The above explanations regarding M1 results in LR- and LT-planes are also valid for M3 since both approaches are identical and produce same results in these planes (Eq. (4) = Eq. (9)). The difference between M1 and M3 lies only in the RT-plane. M3 assumes that the off-axis mechanical properties are distributed in the same way for all planes. Therefore, it also uses Eq. (9) for the RT-plane as it is for LR- and LT-planes.

On the other hand, M1 uses an additional equation (Eq. (5)), which represents a different relation in the off-axis mechanical parameters for the RT-plane (Eq. (1)) in comparison to LR- and LT-planes (Eq. (2)) based on three-dimensional Hankinson's formula in its three-dimensional form. The model for the off-axis mechanical parameters in the RT-plane is described by a sinusoidal function of the angle $\phi$ (Eq. (1) and Fig. 5) [8, 46]. The off-axis parameters in RT-plane are in fact lowest at $\phi=20^{\circ}-30^{\circ}$ and not for $\mathrm{T}$ direction $\left(\phi=0^{\circ}\right)$. This distribution is different compared to the one in LR- and LT- planes, where the lowest parameters are for $\mathrm{R}$ or $\mathrm{T}$ direction, respectively. Clauss et al. [21], who tested wood in-axis and off-axis, has observed this behaviour for the elastic moduli of common ash (Fraxinus excelsior L.) wood. With the adoption of this model in RT-plane, M1 approach accurately estimates the shear moduli for two out of four moisture levels. On the other hand, M3 overestimates the shear moduli for all moisture levels by an average of $30 \%$. The more accurate estimation of the shear moduli by the M1 approach in comparison to M3 might be an indication that the properties of walnut wood RT-plane vary in a similar way as the sinusoidal function of the angle, and comparable with ash wood.

Moreover, the M2 approach (Eqs. (7) and (8)), although different on its assumption for the Poisson's ratios, nearly always provide similar results to the M1 data. This is an indication that Hankinson's formula is also able to estimate the off-axis Poisson's ratio with a reasonable accuracy. On the other hand, the M4 approach (Eq. (10)) shows the highest differences in values compared to the experimental data. In all shear planes and at all moisture content levels, it overestimates the shear moduli by at least $19 \%$ in comparison to the reference data. This may be mainly due to the adopted assumption about the relation of the elastic moduli, which is $E_{i}=E_{j}=\sqrt{E_{i} E_{j}}$. This assumption is invalid for almost all shear planes. In fact, it is only valid for the RT-plane since the $E_{\mathrm{R}} \approx E_{\mathrm{T}}$ [47], which coincides with having obtained the lowest errors for the RT-plane. Although varying for different wood species, the elastic moduli in longitudinal direction is around ten times greater than the one in perpendicular direction. For walnut wood the ratio of elastic moduli of the in-axis directions are $E_{\mathrm{L}}: E_{\mathrm{R}}: E_{\mathrm{T}}=8-12: 1-1.1: 1$ [13], making the above-mentioned assumption not suitable for estimating shear moduli in LR- and LTplane.

While the discrepancies between experimentally obtained and theoretically calculated values can be partly explained by the intrinsic assumptions of the models, inaccuracies may have also been introduced by the experimental techniques (e.g. from specimens size and the presence of notches in the Arcan setup). Values for the shear moduli as obtained from the Arcan tests might deviate from the true shear moduli.

\subsection{Theoretical approaches for estimating the shear strength}

The shear strengths $\left(\tau_{\mathrm{u}}\right)$ obtained from the theoretical approaches and from the mechanical tests are presented in Fig. 7 and in Table S5. The S2 (Strength 2) approach, which is based on combination of Hankinson's formula and the Norris failure criterion, gives the best estimation of shear strengths with average deviations of $-7,-1$ and $-30 \%$ in regard to the values obtained from the shear block tests for LR, LT and RT-plane, respectively. In fact, for the LR and the LT plane, all experimentally derived values are 
correctly estimated by the theoretical approaches as the differences are below the level of significance. Significant errors arise for the RT-plane, which are discussed below. The other approaches, S1 and S3, estimate the shear strengths in LR- and LT-plane with less accuracy giving overestimations of up to $31 \%$ from the shear block data.

The differences in the accuracy of estimating shear strengths between the different approaches mainly originate from their assumption of the failure criteria. The S2 approach incorporates an additional relation between the two perpendicular in-axis strengths, which is not the case for the S1 approach. In the ellipsoidal failure criteria adopted by the $\mathrm{S} 1$ approach, the strengths are taken as independent from each other (Eq. (11)). The S3 approach, on the other hand, does not only assume a certain relationship between the two perpendicular axes but also to the third axis (Eq. (17)). Part of the error obtained for the S3 approach is, thus, also related to the inconsistency of the assumptions made in the Tsai-Hill failure criterion. When LR- and LT- planes are observed, the strength in the perpendicular plane has to be assumed equal $\left(\sigma_{\mathrm{uR}}=\sigma_{\mathrm{uT}}\right)$. Otherwise, Eq. (18) may give an imaginary result due to the square root of a negative value. This assumption, on the other hand, is not true since the strength in $\mathrm{R}$ direction is often higher than in $\mathrm{T}$ direction [8]. In fact, for walnut wood $\sigma_{\mathrm{uR}}$ are in average $20 \%$ higher than $\sigma_{\mathrm{uT}}[14]$.

In RT-plane, all three theoretical approaches, including S2, underestimate the shear strengths by $19-40 \%$ in comparison to the values obtained by the shear block tests. This inaccuracy may also be caused by the invalidity of the shear block test to estimate shear strength in RT-plane as briefly discussed in the previous section. The eccentric loading of the shear block test leads to off-axis stresses (Fig. 4a) in the shear block specimens, which can be represented by in-axis stresses in an orthogonal direction (Fig. 4b). The orthogonal loading consists of a combination of compression stress parallel to the loading, tension stress perpendicular to the loading and shear stress in the tested plane. When the specimen failed, the strength is results of this complex combination of stresses. Due to the structure of wood with its fiber alignment in L-direction, the compressive, tensile and shear loading may equally contribute to the overall loading of the sample for the RT shear plane. To understand the behaviour of wood subjected to a combined stresses, one should consider quadratic wood failure criteria [48]. If the in-axis strength for both $\mathrm{R}$ and $\mathrm{T}$ are assumed equal (Tsai-Hill approach in Tsai, Wu [32]) and the shear strength is smaller than these in-axis strengths $[8,38]$, the calculated results from the theoretical approaches may indeed represent the actual shear strengths.

Overall, the current results show that Norris failure criteria via the S2 approach give the best estimation for shear strength in all planes LR, LT and RT. This finding further confirms the results of Cabrero, Gebremedhin [48], where Norris failure criteria have been proven accurate for wood.

\section{Conclusion}

The moisture dependent shear moduli and shear strengths data of walnut wood (Juglans regia L.) are experimentally obtained in this study using an Arcan test and the shear block test setup. These data combined with the in-axis elastic moduli, Poisson's ratio and strengths data taken from Bachtiar et al. [14] and Bachtiar et al. [13] do now represent the full threedimensional and moisture dependent (at four different moisture levels) data set of walnut wood. Since a good part of historical objects found in the museums is made of walnut wood, its complete data set may contribute to any conservation purposes. The data can serve as input parameters for advance computational modelling of cultural heritage objects by means of finite element methods [49, 50].

Several theoretical approaches for estimating shear moduli and strengths from the in-axis properties are introduced in this study. The shear moduli $(G)$ are estimated from the in-axis elastic moduli $(E)$ and Poisson's ratios $(v)$ while the shear strengths $\left(\tau_{\mathrm{u}}\right)$ are estimated from the in-axis strength $\left(\sigma_{\mathrm{u}}\right)$. While the efficiency of the theoretical approaches to estimate the shear moduli and strengths is undeniable, their results are not always sufficiently accurate. The approaches, which are based on the combination of Hankinson's empirically derived formulas and the tensor rotation give the best results to estimate shear moduli. The shear strengths, on the other hand, are best estimated with the approach based on the combination of Hankinson's formula and Norris' failure criterion. In any cases, when shear properties of an orthotropic material are required, these approaches can be used as 
alternative solutions to the tedious experimental investigations. Besides the experimental test for estimating the shear properties is time-consuming, it may also contain flaws especially for the determination of the shear strength in the perpendicular plane as has been discussed before. The accuracy of these methods has been studied for estimating the shear orthotropic properties of walnut wood. However, further studies are required to proof their accuracy for other wood species and other orthotropic materials.

In almost all cases, the calculated shear moduli and shear strengths obtained from various theoretical approaches are either in agreement (below level of significance) or lower in comparison to the experimentally derived values. From the application point of view, this underestimation of the material properties leads to the less efficient use of the material. On the other hand, this makes the use of the theoretically calculated values acceptable since it will increase the safety factor of the structures against shear failure.

Acknowledgements Special acknowledgement is attributed to Deutsche Forschungsgemeinschaft (DFG, Project No. KA 1163/25) and Swiss National Science Foundation (SNF, Project No. 14762) for the funding of this study. Additionally, we want to thank Thomas Schnider who helped during the specimens preparation.

Funding This work is a part of combined project between TU Dresden, Germany funded by (DFG, Project No. KA 1163/25) and ETH Zurich, Switzerland funded by Swiss National Science Foundation (SNF, Project No. 14762).

\section{Compliance with ethical standards}

Conflict of interest We declare that there is no conflict of interest in this work.

\section{References}

1. Landau LD, Lifshitz EM (1970) Theory of elasticity, vol 7. Course of theoretical physics, 2nd edn. Pergamon Press, Oxford

2. von Mises R (1913) Mechanik der festen Körper in plastisch-deformable Zustand. Nachr d Kgl Ges Wiss Göttingen, Math-phys Klasse 1(1):582-592

3. Tresca H (1864) Mémoire sur l'écoulement des corps solides soumis à de fortes pressions. Imprimerie impériale, Paris

4. Iosipescu N (1967) New accurate procedure for single shear testing of metals. J Mater 2(3):537-566
5. Arcan M, Hashin Z, Voloshin A (1978) A method to produce uniform plane-stress states with applications to fiberreinforced materials. Exp Mech 18(4):141-146. https://doi. org/10.1007/bf02324146

6. Xavier J, Oliveira M, Morais J, Pinto T (2009) Measurement of the shear properties of clear wood by the Arcan test. Holzforschung 63(2):217-225

7. DIN-52187 (1979) Prüfung von Holz: Bestimmung der Scherfestigkeit in Faserrichtung. Beuth Verlag $\mathrm{GmbH}$, Berlin

8. Bodig J, Jayne B (1993) Mechanics of wood and wood composites. Krieger Publishing Company, Malabar

9. Bucur V, Archer R (1984) Elastic constants for wood by an ultrasonic method. Wood Sci Technol 18(4):255-265

10. Müller U, Ringhofer A, Brandner R, Schickhofer G (2015) Homogeneous shear stress field of wood in an Arcan shear test configuration measured by means of electronic speckle pattern interferometry: description of the test setup. Wood Sci Technol 49(6):1123-1136. https://doi.org/10.1007/ s00226-015-0755-3

11. Saint-Venant B (1863) Mémoire sur la destribution d'lasticit. Journal de Mathématiques Pures et Appliquées (Liouville), ser 2, t 8

12. Lekhnitskii S (1963) Theory of elasticity of an anisotropic elastic body. Holden Day, Inc., San Francisco

13. Bachtiar EV, Sanabria SJ, Mittig JP, Niemz P (2017) Moisture-dependent elastic characteristics of walnut and cherry wood by means of mechanical and ultrasonic test incorporating three different ultrasound data evaluation techniques. Wood Sci Technol 51(1):47-67. https://doi.org/ 10.1007/s00226-016-0851-z

14. Bachtiar EV, Rüggeberg M, Niemz P (2017) Mechanical behavior of walnut (Juglans regia L.) and cherry (Prunus avium L.) wood in tension and compression in all anatomical directions. Revisiting the tensile/compressive stiffness ratios of wood. In print Holzforschung 2017

15. DIN-52184 (1979) Prüfung von Holz: Bestimmung der Quellung and Schwindung. Beuth Verlag GmbH, Berlin

16. DIN-52183 (1977) Prüfung von Holz: Bestimmung des Feuchtigkeitsgehaltes. Beuth Verlag GmbH, Berlin

17. Hasanpour R, Choupani N (2009) Rock fracture characterization using the modified Arcan test specimen. Int J Rock Mech Min Sci 46(2):346-354

18. Deshpande V, Fleck N (2001) Multi-axial yield behaviour of polymer foams. Acta Mater 49(10):1859-1866

19. Cognard J-Y, Sohier L, Davies P (2011) A modified Arcan test to analyze the behavior of composites and their assemblies under out-of-plane loadings. Compos A Appl Sci Manuf 42(1):111-121

20. Jurf RA, Pipes RB (1982) Interlaminar fracture of composite materials. J Compos Mater 16(5):386-394

21. Clauss S, Pescatore C, Niemz P (2014) Anisotropic elastic properties of common ash (Fraxinus excelsior L.). Holzforschung 68(8):941-949

22. Keunecke D, Hering S, Niemz P (2008) Three-dimensional elastic behaviour of common yew and norway spruce. Wood Sci Technol 42(8):633-647

23. Hankinson R (1921) Investigation of crushing strength of spruce at varying angles of grain. Tech. Rep. No. 259, U.S. Air Service 
24. Yoshihara H (2009) Prediction of the off-axis stress-strain relation of wood under compression loading. Eur J Wood Wood Prod 67(2):183-188

25. Radcliffe B (1965) A theoretical evaluation of Hankinson's formula for modulus of elasticity of wood at an angle to the grain. Quart Bull Michigan Agr Exp Sta 48(1):286-295

26. Keylwerth R (1951) The anisotropic elasticity of wood and plywood (Die anisotrope Elastizität des Holzes und der Lagenhölzer). Verlag des Vereins Deutscher Ingenieure VDI-Forschungsheft 430

27. Garab J, Keunecke D, Hering S, Szalai J, Niemz P (2010) Measurement of standard and off-axis elastic moduli and poisson's ratios of spruce and yew wood in the transverse plane. Wood Sci Technol 44(3):451-464

28. Kon T (1948) On the law of variation of the modulus of elasticity for bending in wooden beams. Bull Hokkaido Univ Dept Eng 1(1):157-166

29. Aicher S, Klöck W (2001) Linear versus quadratic failure criteria for in-plane loaded wood based panels. Otto-Graft-J 12(1):187-199

30. Norris C (1962) Strength of orthotropic materials sucbhected to combined stresses. Technical Report No. 1816. U.S. Dept. of Agriculture, Forest Service Forest Products Laboratory, Madison, Wisconsin, USA

31. Hill R (1950) The mathematical theory of plasticity. Oxford University Press Inc., New York

32. Tsai SW, Wu EM (1971) A general theory of strength for anisotropic material. J Compos Mater 5(1):58-80

33. Ku HH (1966) Notes on the use of propagation of error formulas. J Res Natl Bureau Stand 70(4):263-273

34. Stephens MA (1974) EDF statistics for goodness of fit and some comparisons. J Am Stat Assoc 69(347):730-737. https://doi.org/10.1080/01621459.1974.10480196

35. Snedecor G, Cochran W (1989) Statistical methods, 8th edn. Iowa University Press, Iowa City

36. Kollmann FFP, Coté WA (1984) Principles of wood science and technology. Springer, Berlin

37. Bucur V (2006) Acoustic of wood. Springer Series in Wood Science. Springer, Berlin
38. Wagenführ A, Scholz F (2012) Taschenbuch der Holztechnik. Carl Hanser Verlag GmbH \& Co. KG, München

39. Reichel S (2015) Modellierung und Simulation hygromechanisch beanspruchter Strukturen aus Holz im Kurzund Langzeitbereich. Technische Universität Dresden, Dresden

40. Ozyhar T, Mohl L, Hering S, Hass P, Zeindler L, Ackermann R, Niemz P (2016) Orthotropic hygric and mechanical material properties of oak wood. Wood Mater Sci Eng 11(1):36-45. https://doi.org/10.1080/17480272.2014. 941930

41. Sonderegger W, Martienssen A, Nitsche C, Ozyhar T, Kaliske M, Niemz P (2013) Investigations on the physical and mechanical behaviour of sycamore maple (Acer pseudoplatanus L.). Eur J Wood Wood Prod 71(1):91-99. https://doi.org/10.1007/s00107-012-0641-8

42. Donaldson L (2011) Delamination of wood at the microscopic scale: current knowledge and methods. In: Bucur V (ed) Delamination in wood, wood products and wood-based composites. Springer, Dordrecht, pp 123-144

43. Gerhards C (1982) Effect of moisture content and temperature on the mechanical properties of wood: an analysis of immediate effects. Wood Fiber Sci 14(1):4-36

44. Skaar C (1988) Wood-water relations. Springer, New York

45. Winandy JE, Rowell RM (1984) The chemistry of wood strength. ACS Publications, Washington

46. Goodman JR, Bodig J (1971) Orthotropic strength of wood in compression. J Wood Sci 4(2):83-94

47. Ross RJ (2010) Wood handbook: wood as an engineering material. U.S. Dep. Agric, Madison

48. Cabrero JM, Gebremedhin KG (2010) Evaluation of failure criteria in wood members. In: WCTE 2010: World Conference on Timber Engineering, Riva Del Garda, Trento, Italy

49. Zítek P, Vyhlídal T (2009) Model-based moisture sorption stabilization in historical buildings. Build Environ 44(6):1181-1187

50. Konopka D, Gebhardt C, Kaliske M (2016) Numerical modelling of wooden structures. J Cult Herit 24:S93-S103 